\title{
Arnold Esch
}

\section{Ein Sonderfall deutscher Präsenz in Rom: Die erste Generation deutscher Frühdrucker nach vatikanischen Quellen}

Unter den im spätmittelalterlichen Italien, und zumal in Rom, massenhaft anzutreffenden Deutschen sei hier ein Sonderfall vorgeführt: die deutschen Frühdrukker in Rom. Ein Sonderfall insofern, als ihr Metier sich von den durch Deutsche in Rom bevorzugt ausgeübten Gewerben - sie waren Bäcker, Schuster, Wirte, Söldner, Bauarbeiter - einigermaßen unterschied. Und sie waren ein besonderer Fall auch in zeitlicher, sozusagen konjunktureller Hinsicht: Niemals stärker als im 3. Viertel des 15. Jahrhunderts - in der ersten und zweiten Generation nach Erfindung des Buchdrucks - konnten die deutschen Buchdrucker so sicher darauf zählen, in der Fremde willkommen zu sein und sich auch ohne viel Eigenkapital dort etablieren zu können. Sie waren alle nicht als Drucker geboren, waren alle älter als die Erfindung ihrer Kunst, und so wagten sie sich - und das fern der Heimat - auf völlig unbekanntes Terrain. Aber sie durften zuversichtlich sein. Zwar würde es in Rom nicht genügen, in der nächsten Klosterscheune eine alte Weinpresse aufzustellen und das Handgießinstrument auszupacken. Aber dafür durften die, die um das Jahr 1465 in Rom und Subiaco auftauchten, neugieriges Interesse und großen Bedarf, kurz: einen Markt, erwarten.

Ich will im folgenden nicht wiederholen, was man über die ersten deutschen Drucker in Rom ${ }^{1}$ weiß, sondern einiges Material, das in den letzten Jahren bei der systematischen Durchforschung der vatikanischen Bestände für das Repertorium Germanicum neu zutage getreten ist und das ich im Gutenberg-Jahrbuch 1993 bekannt gemacht habe ${ }^{2}$, für die Zwecke dieser Tagung kurz interpretieren. Die Provenienz aus den vatikanischen Registern führt dabei auf gewisse Aspekte, die bis-

1 Vor allem Konrad Haebler, Die deutschen Buchdrucker des XV. Jahrhunderts im Auslande (München 1924); Ferdinand Geldner, Die deutschen Inkunabeldrucker, Bd. 2: Die fremden Sprachgebiete (Stuttgart 1970); Scrittura, biblioteche e stampa a Roma nel Quattrocento. Aspetti e problemi. Atti del seminario 1-2 giugno 1979, a cura di Concetta Bianca, Paola Farenga, Giuseppe Lombardi, Antonio G. Luciani e Massimo Miglio (Città del Vaticano 1980); Atti del secondo seminario 6-8 maggio 1982, a cura di Massimo Miglio (Città del Vaticano 1983); weitere Literatur in den in Anm. 2-4 und 8 genannten Artikeln.

2 Arnold Esch, Deutsche Frühdrucker in Rom in den Registern Papst Pauls II., in: Gutenberg-Jahrbuch (1993) 44 ff. (im folgenden um einige Nachträge ergänzt). 
her nicht so sehr beachtet wurden, für unsere Fragestellung - deutsche Frühdrukker nicht irgendwo, sondern im Zentrum der Weltkirche - aber nicht unwichtig sind: den Kleriker-Status vieler Drucker, ihre Erwartungen gegenüber der Institution Kirche, ihr Interesse am Pfründenmarkt. Ja, in den vatikanischen Registern bekommen wir einige dieser Personen zu fassen, noch bevor sie in Rom und noch bevor sie überhaupt Frühdrucker sind. Und das ist für Herkunft und Lebensbedingungen einer ersten Generation doch von Bedeutung.

Zunächst aber einige neuere Nachrichten aus nichtvatikanischen römischen Archivalien. Die Durchsicht römischer Notarsimbreviaturen förderte jüngst zwei frühe Gesellschaftsverträge von deutschen Druckern in Rom zutage. Der eine Fall ist bemerkenswert wegen seines Datums: terminus ante ist der Herbst 1466 (als Pannartz und Sweynheym noch gar nicht in Rom, sondern in Subiaco arbeiteten): diese sotietas super inpressione librorum conficiendorum cum formis nennt unter den Gesellschaftern einen Sohn des deutschen Erstdruckers in Rom Ulrich Han. In der anderen Gesellschaft, sotietas ad condendum et conficiendum libros cum formis mit dem bemerkenswerten Gesellschaftskapital von 1200 duc., verbanden sich 1469 zwei Deutsche, darunter ein Goldschmied, mit Italienern, darunter wiederum Goldschmieden (die sich nämlich am ehesten auf das Schneiden der Typen verstanden) und einem Kurialen, einem cubicularius pape; dabei sollte das Berufsgeheimnis nicht allen Gesellschaftern eröffnet werden müssen (non teneantur... ostendere aliquod secretum artis) ${ }^{3}$.

In römischen Notarsimbreviaturen fanden sich auch weitere deutsche Frühdrucker, die der Forschung bisher nicht bekannt waren: so ein magister Raynoldus de Colonia impressor librorum und ein magister Orrigus de Vrenda impressor librorum als Teilnehmer einer Versammlung der Bruderschaft des Campo Santo Teutonico 1474, sowie ein magister Johannes Fersoris magister librorum ad forma clericus Erbipolensis 1473 mit einem ungewöhnlichen Mietvertrag, aufgesetzt von dem in Rom tätigen deutschen Notar Johannis Michaelis: Johannes mietet auf $1 \mathrm{Jahr} 3$ Pressen, tria torcularia apta ad imprimendum libros cum omnibus rebus necessariis ad ea preterquam litteris, also mit aller Ausstattung außer den Typen. Und er zahlt dafür nicht in Geld, sondern in Produkten: quinque volumina pro quolibet torculari pro quolibet libro per eum imprimendo, „5 Bände für jede Presse von jedem gedruckten Buch". Vermieterin ist die Frau jenes Clemente Donati von Urbino, der 1470, vergeblich, Borso d'Este die Einführung des Buchdrucks in Ferrara vorgeschlagen hatte ${ }^{4}$.

3 Anna Modigliani, Tipografi a Roma prima della stampa (Roma nel Rinascimento, Inedita 3, Roma 1989).

${ }^{4}$ Archivio di Stato di Roma, Notai Capit. 1134 fol. 224rv (der Notar Johannes Michaelis, über den ich demnächst einen Beitrag vorlegen werde): vgl. Paolo Cherubini, Anna Esposito, Anna Modigliani, Paolo Scarcia Piacentini, Il costo del libro, in: Scrittura (wie Anm. 1) Bd. 2, $422 \mathrm{f}$. (Johannes Fersoris ist im Repertorium Germanicum um 1473 nicht nachzuweisen). Auch Hans von Lande[n]bach unter die deutschen Frühdrucker in Rom einzureihen (so der Vorschlag von J. L. Flood, in: The Italian Book 1465-1800. Studies presented to Dennis E. Rhodes, ed. by Denis V. Reidy, London 1993), ist mit der Heidelberger Grabplatte von 1514 
Die andere römische Quellengattung, die bisher wenig beachtet wurde und nun gleichfalls Nachrichten über Buchhandel und Frühdrucker in Rom hergab, sind die Zollregister, die gerade für die hier interessierenden Jahrzehnte erhalten sind ${ }^{5}$. $\mathrm{Da}$ werden im Januar 1468 in einer Lieferung 60 Exemplare von Augustins De civitate Dei vom Kloster Subiaco durch den römischen Zoll gebracht: Das ist der von Pannartz und Sweynheym damals im Kloster Subiaco hergestellte Druck, der früheste in Italien überhaupt. Auffallend ist, wie viele der nach Rom hineinkommenden Buchlieferungen unter dem Namen von Deutschen laufen: Vito tedesco, Stefano tedesco (vielleicht die deutschen Frühdrucker Vitus Puecher und Stephan Planck), usw. - noch in der zweiten Jahreshälfte 1474 werden 18 von 45 Büchersendungen von Deutschen deklariert. Bei den Importen zu Schiff läßt sich das weniger leicht erkennen. Jedenfalls sind auch Frühdrucke darunter, denn der Hafenzoll vermerkt bisweilen ausdrücklich: libri da stampa. Im Juli 1474 werden sogar zwei Druckerpressen am Tiberhafen ausgeladen, sozusagen rechtzeitig zum Heiligen Jahr 1475 mit seiner zu erwartenden erhöhten Nachfrage: 2 stromenti che sse operano da fare libri de stampa, „zwei Instrumente, die man gebraucht, um gedruckte Bücher herzustellen"!

Aber nun zu vatikanischen Registern und ihren Nachrichten über deutsche Frühdrucker in Rom, zumal es bei diesem Colloquium ja mehr um die Personen und ihre Lebensbedingungen als um die Produkte ihres Handwerks, mehr um die Frühdrucker als um den Frühdruck geht. Dabei seien nicht so sehr einzelne Personen, sondern gewisse gemeinsame Züge behandelt, die für Frühdrucker in Rom typisch sein könnten.

Von bedeutenden Inkunabelforschern ist die Ansicht geäußert worden, daß „Drucker, die aus dem geistlichen Stande hervorgegangen waren“, in der Regel "ihre Schritte nach Rom gerichtet“ hätten, oder daß ein „Kleriker ... nur so lange als Buchdrucker tätig [war], bis er eine kirchliche Pfründe erhielt"6. Diese Meinung dürfte sich im Lichte des Repertorium Germanicum, das normales Verhalten von Klerikern am Pfründenmarkt leichter erkennen läßt, so nicht aufrechterhalten lassen. Warum sollte ein gewöhnlicher Kleriker nach Rom gehen, wo er alles andere als ein König war? Und warum sollte er ein solch spezielles, anspruchsvolles Gewerbe wie den Buchdruck wegen einer Pfründe aufgeben, wo doch viele der beantragten Pfründen ihn gar nicht zur Anwesenheit vor Ort verpflichteten?

Zunächst einmal: Daß unter den Frühdruckern nicht wenige Kleriker waren, ist von der Forschung immer schon beachtet worden und mit der Nähe dieses Metiers zum Umgang mit Texten, ja zum intellektuellen Milieu erklärt worden. Nun zeigt sich aber aus dem vatikanischen Material, daß unter den deutschen Frühdruckern in Rom auch solche waren, die als Laien nach Rom kamen und erst dort

schwer zu begründen: Die ersten bücher truckt ich zu Rom meint wohl nur: meine ersten Bücher druckte ich in Rom.

5 Verzeichnis importierter Bücher in Scrittura (wie Anm. 1) Bd. 2, 538ff.; vgl. Arnold Esch, Importe in das Rom der Renaissance. Die Zollregister der Jahre 1470 bis 1480, in: Quellen u. Forsch. aus ital. Archiven u. Bibl. 74 (1994) 393 ff., 410f., 425; ders., (wie Anm. 2) $45 \mathrm{f}$.

${ }^{6}$ Haebler, (wie Anm. 1) 64 bzw. Geldner, (wie Anm. 1) 37. 
den Klerikerstatus erwarben: Konrad Sweynheym ist, anders als Haebler annahm, in Subiaco und Rom zunächst anscheinend noch Laie, und auch der Frühdrucker Georg Sachsel erwirbt die ersten Weihegrade nachweislich erst in Rom?

Ein wichtiger Beweggrund zu solchem Schritt war vermutlich auch die verlokkende Aussicht, sich so, und nur so, um Pfründen bewerben zu können (und auf diesen Geschmack konnte man in Rom wohl kommen): Für den Fall unternehmerischen Mißerfolgs, oder bei der Rückkehr in die Heimat, bot das doch eine gewisse Sicherung. Pannartz und Sweynheym kamen bekanntlich, wie ihre vielbehandelte Bittschrift an Papst Sixtus IV. mit detaillierter Angabe der unverkauften Auflagen zeigt, schon bald in wirtschaftliche Schwierigkeiten, weil der Markt mit klassischen Autoren vorläufig gesättigt war und man sich Titel von dauerhafterer Nachfrage (Kanonistisches, Consilia-Sammlungen, Ablaßverzeichnisse usw.) einfallen lassen mußte ${ }^{8}$. Tatsächlich beginnt Sweynheym, 1469 wohl noch Laie, spätestens 1471 in der Krise, sogleich mit mehreren Anträgen an den Pfründenmarkt zu gehen. Damit sollen echte Gefühle persönlicher Berufung bei der Erwerbung der niederen Weihen natürlich nicht ausgeschlossen werden. Aber wenn Sweynheym im gleichen Jahr zwei beliebige Kanonikate, eine Vikarie im Dom von Speyer und eine Pfarrei in Jeserigk beantragte und bewilligt erhielt, dann hatte er gewiß nicht vor, nach Speyer zu gehen oder Pfarrer im Brandenburgischen zu werden (für diese Pfarrei hätte er auch einen Vikar bestellen und die Differenz einbehalten, oder er hätte sie gegebenenfalls gegen eine Pension resignieren können): Er versuchte nur einfach an kirchliche Einkünfte zu kommen (gerade darum bewarb man sich ja um Benefizien sine cura), blieb aber in Rom, wo er 1477 starb. Und wenn Adam Rot, wie seine Suppliken zeigen, es auf mehrere weit auseinanderliegende Benefizien in den Diözesen Basel, Straßburg und Metz absah, so zeigt allein schon diese breite Streuung, daß an persönliche Präsenz nicht gedacht war, sondern daß Rot ins Pfründengeschäft einsteigen wollte, um seinen Pfründbesitz eventuell später an irgendeiner Stelle durch Tausch zu verdichten ${ }^{9}$. Um seine Pfarrei behalten zu können, ohne sie persönlich versehen zu müssen, erwirkte er denn auch den Aufschub seiner Priesterweihe auf die längste nach kanonischem Recht statthafte Frist von 7 Jahren und druckte seine Bücher in Rom munter weiter.

Und so machten es auch die anderen deutschen Frühdrucker in Rom, ja erstaunlicherweise sieht man, wie nicht weniger als sechsen von ihnen am gleichen Tag ihr Pfründenantrag genehmigt wird, mit dem sie sich um je zwei Kanonikate und zwei Pfruinden beliebiger Kollatoren bewarben: Arnold Pannartz und Konrad Sweynheym, Sixtus Rüssinger und Adam Rot, Georg Sachsel und Bartholo-

7 Esch, (wie Anm. 2) unter den Namen; auf die dortigen Belegstellen wird fortan nicht mehr im einzelnen verwiesen.

${ }^{8}$ Neueste Literatur: Edwin Hall, Sweynheym and Pannartz and the Origins of Printing in Italy. German Technology and Italian Humanism in Renaissance Rome (McMinnville 1991); Gutenberg e Roma. Le origini della stampa nella città dei papi (1467-1477), a cura di Massimo Miglio e Orietta Rossini (Napoli 1997).

9 Zu System und Praxis knapp und kompetent Brigide Schwarz, Römische Kurie und Pfründenmarkt im Spätmittelalter, in: Zeitschrift für historische Forschung 20 (1993) $129 \mathrm{ff}$. 
meus Golsch, sie alle am 1. Januar 147210! Das heißt nun nicht, daß sie alle tatsächlich an diesem Tag im Vatikan vorstellig wurden; es besagt vielmehr, daß sie alle in der Lage waren, ein sogenanntes „Vorzugsdatum“ zu ergattern, das bei konkurrierenden Anträgen die bessere Ausgangsposition verschaffte - und das „beste“ Datum ${ }^{11}$ im Pontifikat Sixtus' IV. war eben dieser 1. Januar 1472.

Damit berühren wir einen nächsten Aspekt, der für unser Thema wichtig ist: das Ansehen dieser ausländischen Buchdrucker in ihrer ersten römischen Generation. Indizien dafür lassen sich in den Supplikenregistern nur ersehen, wenn man die „normale“ Praxis der Pfründenvergabe - und die nicht jedermann erreichbaren Prärogativen bevorzugter Berücksichtigung kennt: Neben dem Vorzugsdatum ist die Kategorie der (erbetenen und dann auch bewilligten) Pfründenanwartschaften ein solches Indiz für Ansehen bzw. Bevorzugung, ebenso ein motu proprio, und auch die Gratis-Expedition erhält nicht jeder. Daß Sweynheym 1469 für seine Pfarrkirche im heimatlichen Schwanheim ${ }^{12}$ einen Ablaß zu erwirken vermag, erwartet man eher bei einem Fürsten oder einem Adeligen als bei einem gewöhnlichen Laien - aber als librorum in alma Sanctitatis Vestre Urbe impressor (so die Supplik) war man offensichlich nicht "gewöhnlich“, verschaffte Leistung Nähe zur Kurie.

Kurien-Nähe ergibt sich ganz explizit auch aus Status-Angaben. Erst seit neuem wissen wir, daß Ulrich Han, der Erstdrucker in Rom (und Italien), schon 1466 Familiar des Papstes war: Sein römischer Erstdruck datiert vom Ende dieses Jahres. Bartholomeus Guldinbeck war schon 1469 Familiar des Kardinals Amico Agnifili (und zwar einer der 12 bevorzugten, nämlich der sogenannten familiares descripti, die bei der Pfründenvergabe Vorrechte genossen). Seine datierten römischen Drucke beginnen erst 6 Jahre später.

In beiden Fällen muß man davon ausgehen, daß diese Deutschen wohl schon einige Zeit in Rom lebten, bevor sie hier mit dem Drucken begannen. Und das führt auf einen weiteren Punkt. Denn auch bei Georg Sachsel wissen wir nun mit Sicherheit, daß er schon Jahre vor seinem ersten datierten Druck (1474) in Rom war, da er sich den Empfang von Tonsur und ersten vier Weihegraden im Lateran 1466 ausdrücklich bestätigen läßt; und auch bei Sixtus Rüssinger läßt sich frühere Ankunft in Rom wenigstens vermuten. Mit anderen Worten: Sie waren vielleicht gar nicht als Buchdrucker nach Rom gekommen, sondern erst in Rom dazu geworden. Doch mag es auch sein, daß sie zunächst in der Druckerei von Pannartz und Sweynheym gearbeitet und ihr Metier gelernt hatten, bevor sie unter eigenem Namen zu drucken begannen. Denn bei Adam Rot und bei Georg Sachsel haben

10 Esch, (wie Anm. 2) 47.

11 Anfangs beispielsweise das Krönungsdatum, jetzt ein fiktives, in den Kanzleiregeln des jeweiligen Papstes festgelegtes Datum für die bevorzugte Bewilligung zahlreicher Suppliken: dazu Andreas Meyer, Spätmittelalterliches Benefizialrecht im Spannungsfeld zwischen päpstlicher Kurie und ordentlicher Kollatur: Forschungsansätze und offene Fragen, in: Monumenta Iuris Canonici C 9 (1992) bes. $252 \mathrm{ff}$.

12 Wegen des Patroziniums wohl nicht Schwanheim bei Höchst, sondern vielleicht Schwanheim an der Bergstraße: s. Esch, (wie Anm. 2) Anm. 23. 
die von ihnen verwendeten Typen immer schon Nähe zu dieser Werkstatt vermuten lassen, und wenn man den enormen, von Haebler auf 5000 Seiten pro Tag berechneten Ausstoß dieser Presse bedenkt, müssen Pannartz und Sweynheym tatsächlich viele Mitarbeiter gehabt haben. Und auch jener clericus Erbipolensis, der sich dann im Rom 3 Druckerpressen mietete, wird sein Metier eher in Rom als in Würzburg erlernt haben.

Vertrautheit mit Rom, Familiarenstatus, Ansehen aufgrund ihres Handwerks: All das (und nicht etwa der Klerikerstatus als solcher, der nur notwendige, nicht hinreichende Voraussetzung war) erklärt nun aber auch ihre eifrige, weil aussichtsreiche Bewerbung um Pfründen. Als Rüssinger sich um eine Pfründe im Elsaß bewirbt, weiß er, daß sie binnen kurzem frei sein wird (vacatura, sagt seine Supplik), nämlich durch den Verzicht eines deutschen Familiaren des Kardinals Todeschini-Piccolomini - so wie, umgekehrt, beim Tode von Arnold Pannartz im Frühjahr 1476 ein wohlinformierter Kölner Kleriker und Papstfamiliar sofort seine Anwartschaft auf die (zwischen Pannartz und ihm strittige) Altarvikarie in Köln betrieb ${ }^{13}$. Und Sweynheym weiß, daß die gewünschte Vikarie in Speyer vakant geworden ist durch den Tod eines deutschen Papstfamiliaren - auch das ein Indiz, daß diese Drucker durch ihre Kurien-Nähe und durch ihre Kontakte in der deutschen Kolonie einen Informations-Vorsprung hatten, den sie zu nutzen wußten. Und wenn derselbe Sweynheym auf eine Pfründe schon nach 6 Tagen (!) zugunsten eines anderen verzichtete, so zeigen solch seltsame Manöver, daß er beim Spiel am Pfründenmarkt schon bald einige Virtuosität entwickelte.

13 Archivio Segreto Vaticano, Reg. Suppl. 737 fol. 194v, 18.4.1476 und fol. 254r, 25.4.1476 (freundlicher Hinweis von Dr. Juliane Trede). Da sein letzter datierter Druck vom 28. März 1476 ist (Geldner II, 44), läßt sich nun auch das Todesdatum von Pannartz eng eingrenzen. 